\title{
Parallel and Passive Distribution to Arrayed Microwells Using Self-Regulating Pinched Flow
}

\author{
Kentaro Kawai ${ }^{*}$, Masaki Kanai ${ }^{1}$, Tatsuya Munaka ${ }^{1}$, \\ Hirohisa Abe ${ }^{1}$, Akira Murakami² and Shuichi Shoji ${ }^{3}$ \\ Major in Nanoscience and Nanoengineering, Waseda University, \\ 3-4-1 Okubo, Shinjuku-ku, Tokyo 169-8555, Japan \\ ${ }^{1}$ Technology Research Laboratory, Shimadzu Corporation, \\ 3-9-4 Hikari-dai, Seika-cho, Soraku-gun, Kyoto 619-0237, Japan \\ ${ }^{2}$ Graduate School of Science and Technology, Kyoto Institute of Technology, \\ Matsugasaki, Sakyo-ku, Kyoto 606-8585, Japan \\ ${ }^{3}$ Faculty of Science and Engineering, Waseda University, \\ 3-4-1 Okubo, Shinjuku-ku, Tokyo 169-8555, Japan
}

(Received June 27, 2008; accepted September 12, 2008)

Key words: passive distribution, parallel distribution, microwell array, self-regulating pinched flow, high throughput screening, cellular diagnostics

A parallel and passive (without any actuators or electrodes) cell distribution method for a cellular diagnostic microwell array was developed. This method can be used to simultaneously distribute cells evenly to arrayed microwells only by introducing cell suspensions into the inlet. To regulate the dispersion of the number of distributed cells into each microwell, we conceived a novel concept, the self-regulating pinched flow. The self-regulating pinched flow is realized in a functional microchannel, which consists of center micropillar colonnades and side channels. Computational fluid dynamics (CFD) simulations were carried out to optimize the design of the pinching area. We developed a prototype device that can be used to successfully distribute the beads uniformly in eight parallel microwells. The coefficient of variation (CV) of the distributed beads in the microwells was $29.3 \%$.

\section{Introduction}

In the field of biological analysis, micro-total analysis systems ( $\mu$ TASs) have attracted considerable interest. $\mu$ TASs have significant advantages compared with conventional laboratory instruments as a result of their small size. Because of their small size, $\mu$ TASs can reduce the consumption of sample cells and reagents, as well as reaction time. Because of their disposability, they can prevent biohazard contamination. ${ }^{(1)}$

*Corresponding author: e-mail: kawai@shoji.comm.waseda.ac.jp 
A $\mu$ TAS device for cell analysis is promising for drug screening and toxicity tests in drug discovery. In this field, throughput time and reduction of sample volume are important issues. It is possible to reduce the time required for analysis by arraying the microwells. ${ }^{(2)}$ To reduce the total time for the analysis, the time necessary for introducing cells must also be reduced. In previous research, several methods of serial cell sorting/ distributing have been reported: laminar flow control, ${ }^{(3,4)}$ thermosensitive hydrogels, ${ }^{(5)}$ dielectrophoresis (DEP), ${ }^{(6,7)}$ and valve control. ${ }^{(8)}$ However, all these methods require external, complicated setups to control the cell flow, and the time necessary for introducing cells increases with the number of arrayed microwells. In a previous report, ${ }^{(2)}$ we presented the first prototype of a cellular diagnostic microwell array using a passive distribution method. This method achieved parallel cell distribution in a number of microwells with a simple channel configuration of multistep T-shaped bifurcations. However, in this prototype, the number of distributed cells deviated largely as a result of biased cell flow in the loading channel, as shown in Fig. $1 .^{(9)}$ The coefficient of variation (CV) of this method using eight microwells was $94.9 \%$. At the downstream of the bifurcation,

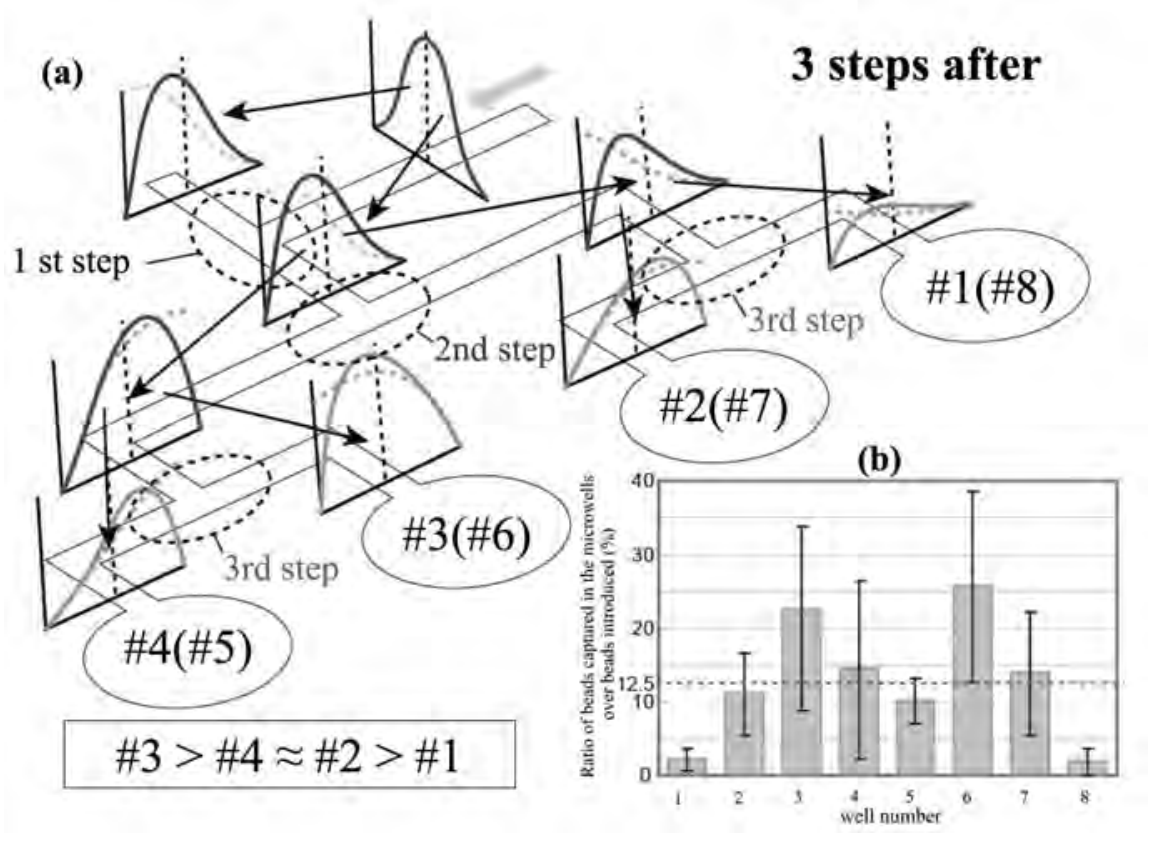

Fig. 1. Results of simple channel configuration in our previous cellular analysis device: (a) bead flow profiles in each branch of the preliminary configuration. When the uniform cell suspension is introduced to the first bifurcation, the flow profile before the first bifurcation becomes symmetric and cells are distributed equally to each branch. However, because the flow profile of the laminar flow is a quadratic curve, the number of the cells that flowed in the center of the channel before the first bifurcation is larger than that of those that flowed in the side of the channel. Therefore, the flow profile after the first bifurcation does not become symmetric and cells are biased to the underside of the channel. This effect is amplified in the second and third bifurcations. (b) Ratio of the number of beads captured in the 8 microwells to the number of beads introduced to the prototype device, averaged over 10 measurements. 
the laminar flow biased the cell flow toward the vertical wall. This biased cell flow caused asymmetric distribution, and this asymmetry was amplified at each step of the bifurcations. To solve this problem, we developed a novel concept of self-regulating flow by applying the pinched flow concept. ${ }^{(10)}$

\section{Concept}

Figure 2 shows the self-regulating pinched structure. At the heart of this structure is the self-regulating pinched flow concept, which regulates the biased cell flow automatically and passively to become a center-aligned cell flow. The self-regulating pinched flow is generated in the front part of the self-regulating pinched structure, which consists of center micropillar colonnades and side channels. Cells are regulated by the self-regulating pinched flow in the pinching area in the rear part. This structure is inserted into the main channel after the T-shaped bifurcation to correct the cell flow that is biased toward the underside by laminar flow. In this structure, the cell flows between the micropillars while most of the carrier flows out between the pillars to the side channels. Since the upper and lower carrier flows in the side channels are pinched at the outlet, the cells are expected to be focused at the center of the outlet. However, when the pinching area is symmetric, almost all of the cells flow out to the lower outlet as shown in Fig. 2(a) because of the biased cell flow generated at the T-shaped bifurcation and the laminar flow in the microchannel. The use of an asymmetric pinching area corrects the cell flow, which is biased toward the underside of the micropillar colonnades, and uniform distribution is obtained, as shown in Fig. 2(b). Computational fluid dynamics (CFD) simulations were carried out to optimize the shape and size of the pinching area.
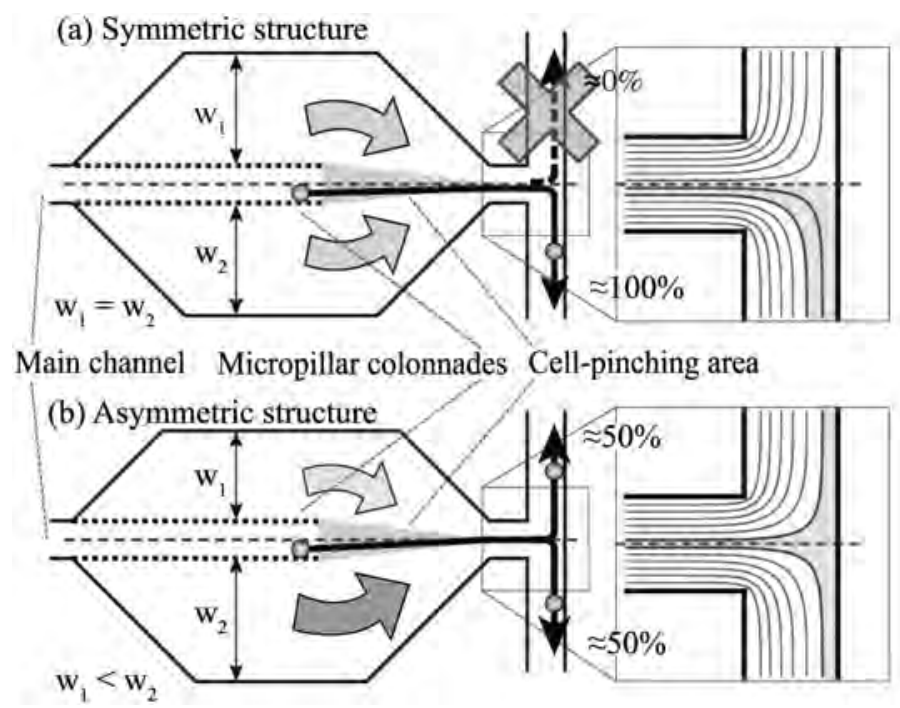

Fig. 2. Schematic illustration of the self-regulating pinched flow: (a) symmetric structure, (b) asymmetric structure. 


\section{Experimental Methods}

A test device for confirming the effect of the self-regulating pinched structure and a prototype device with eight microwells were fabricated on $200-\mu m$-thick Si wafers. The silicon substrate was prepared by deep reactive ion etching (RIE; Surface Technology Systems, U.K.) from both sides to form all the channels and microwells. The width and depth of all the main channels were both $50 \mu \mathrm{m}$.

The test device has a T-shaped bifurcation for generating biased cell flow, followed by two self-regulating pinched structures and two widened microchannels, and finally, by two T-shaped bifurcations to count the number of beads that flow into each of the two branches. The widened microchannels were formed just downstream of the selfregulating pinched structure to evaluate the effect of the focusing. The widened microchannels are $2000 \mu \mathrm{m}$ in length and $1000 \mu \mathrm{m}$ in width.

Figure 3(a) shows a schematic of our prototype device with self-regulating pinched structures after each bifurcation. The self-regulating pinched structure is $700 \mu \mathrm{m}$ in
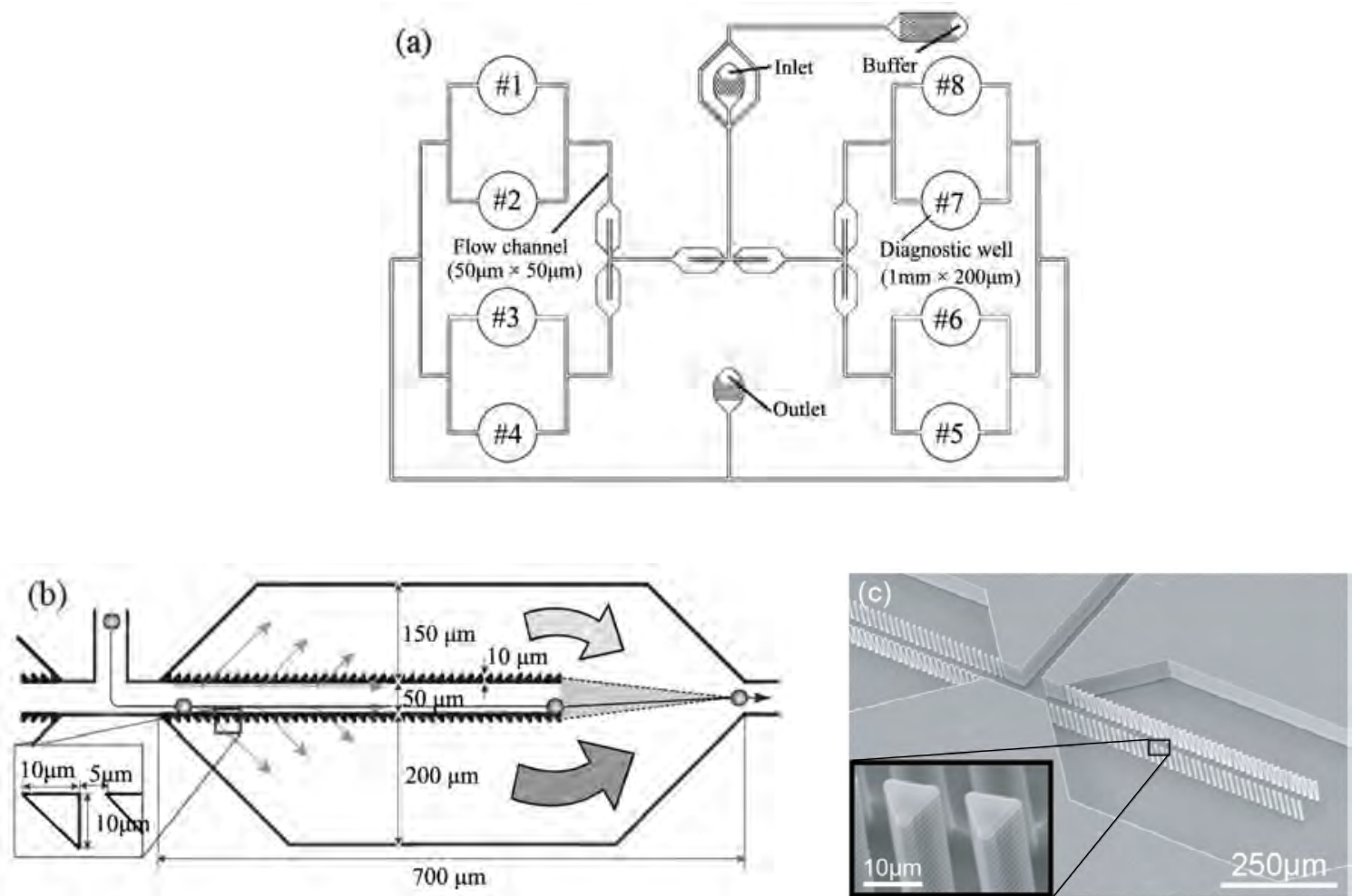

Fig. 3. Different views of our prototype device: (a) schematic design, (b) enlarged view of the self-regulating pinched structure, and (c) SEM images of the self-regulating pinched structure. 
length and $400 \mu \mathrm{m}$ in width, as shown in Fig. 3(b). Double line micropillars of isosceles right triangles ( $10 \mu \mathrm{m}$ in two short sides) with $5 \mu \mathrm{m}$ intervals provide flow channels for the cells of $50 \mu \mathrm{m}$ width. The asymmetric widths of the side channels are 150 and 200 $\mu \mathrm{m}$. The microwells are $1000 \mu \mathrm{m}$ in diameter and $200 \mu \mathrm{m}$ in depth. Scanning electron microscope (SEM) images of the self-regulating pinched structure are shown in Fig. 3(c).

To encapsulate the channels and microwells, Pyrex glass ${ }^{\circledR}$ plates (Corning, U.S.A.) were anodically bonded on both sides of the Si substrate. The motion of the fluorescent beads was observed using a fluorescent microscope (IX71; Olympus, Japan). The images of fluorescent bead trajectories were captured using a three charge-coupled device (3CCD) camera (JK-TU53H; Toshiba, Japan) and then analyzed on a PC using LabView ${ }^{\circledR}$ (National Instruments, U.S.A.). The bead suspension was continuously introduced into the main channel using syringe pumps (KDS200; KD Scientific, U.S.A.).

\section{Results}

In a preliminary evaluation using the test device, a fluorescent microscope and a suspension $\left(1.20 \times 10^{9}\right.$ beads $\left./ \mu \mathrm{L}\right)$ of 1 - $\mu$ m-diameter fluorescent polystyrene beads (Fluorescent polymer microspheres R0100, Duke Scientific, U.S.A.) were used to observe a fluorescent image of the flow stream in the pinching area, as shown in Figs. 4(a) and 4(b). The CFD simulation of the self-regulating pinched flow structure is shown in Fig. 4(c). The two flow streams show the same streamline. These results confirmed the existence of a self-regulating pinched flow. Using a suspension $\left(1.14 \times 10^{4}\right.$ beads $\left./ \mu \mathrm{L}\right)$ of 20- $\mu \mathrm{m}$-diameter fluorescent polystyrene beads (Fluoresbrite ${ }^{\circledR}$, Yellow Green $20.0 \mu \mathrm{m}$ microspheres; Polyscience, U.S.A.) as a substitute for biological cells, we evaluated the passive distribution of the cells by the self-regulating pinched structure. The motions of the beads in the self-regulating pinched structure were captured, as shown in Fig. 5(a). These results show that the beads flowed along the underside of the pillar colonnades and were focused toward the center at the outlet of the self-regulating pinched structure. In the test device, if the focusing was inadequate, the laminar flow would shift the trajectory of the fluorescent beads to the sidewall in the wide test microchannel. As shown in Fig. 5(b), all the fluorescent beads flowed to the center of the test microchannel. This

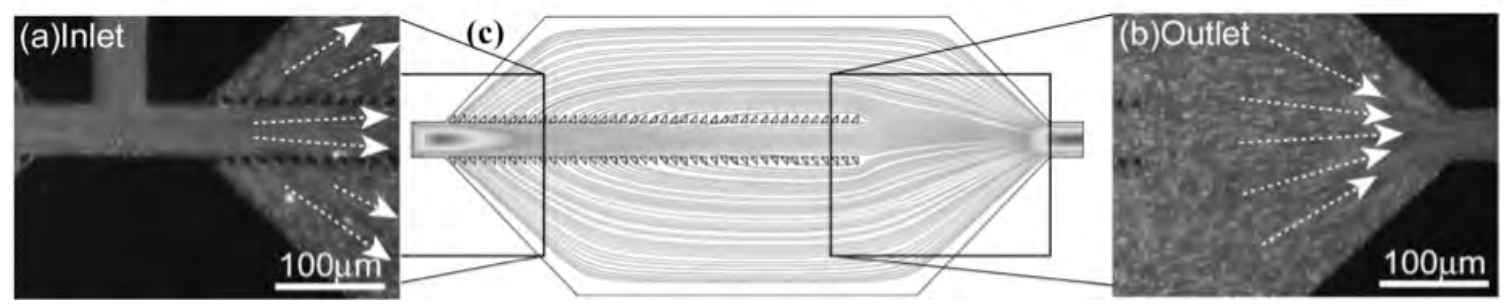

Fig. 4. Streamlines in the self-regulating pinched structure: (a) and (b) are CCD images, (c) CFD simulation results. 
(a)
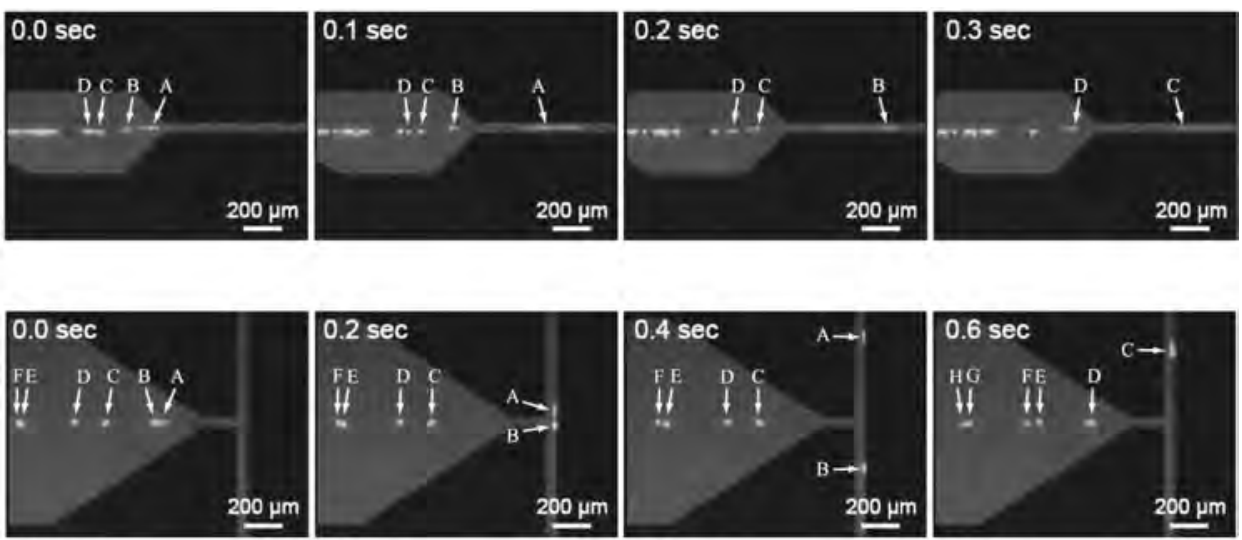

(b)
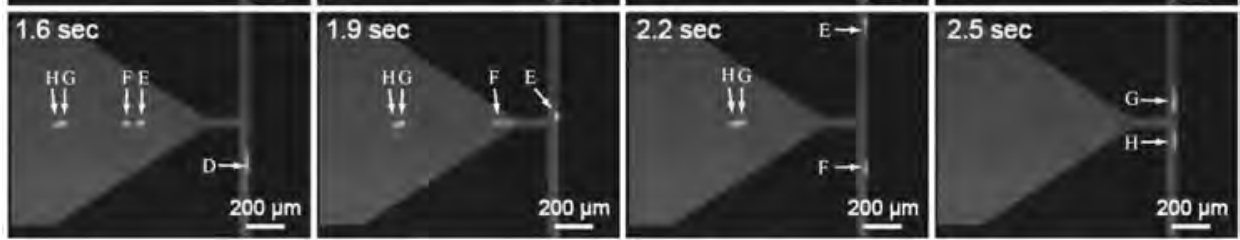

Fig. 5. Optical microscope images of flowing fluorescent beads: (a) in the self-regulating pinched structure and (b) in the widened channel after the self-regulating pinched structure.

result showed that focusing by the self-regulating pinched structure had been optimized. Four hundred and twenty-two fluorescent beads were introduced into the inlet of the asymmetric version of the self-regulating pinched flow structure. Two hundred and fifteen beads flowed to the right outlet, while two hundred and seven beads flowed to the left $(50 \% \pm 0.9 \%)$.

Using our prototype device, which has eight microwells, about 100 fluorescent beads were introduced per measurement over a series of 10 measurements. The suspension of fluorescent beads was introduced from the inlet at a flow rate of $0.8 \mu \mathrm{L} / \mathrm{min}$, and the number of beads distributed into each microwell was measured. Optical microscope images showing fluorescent bead distributions are shown in Fig. 6. Parallel and passive distribution was carried out and the fluorescent beads were distributed amongst the microwell. The average distribution in each microwell over 10 test measurements is shown in Fig. 7. The CV of the average of eight microwells was 29.3\%. It is clear from this result that the uniformity of bead distribution among the eight microwells was improved compared with that of the simple T-shaped configuration.

\section{Discussion}

Through the experimental evaluation using a fluorescent beads suspension, we confirmed the feasibility of the passive distributing method. For practical use with cells and culture media, there is a problem of channel clogging. Since the passive distributing method is based on the equal flow conductance of each channel, the clogging causes 


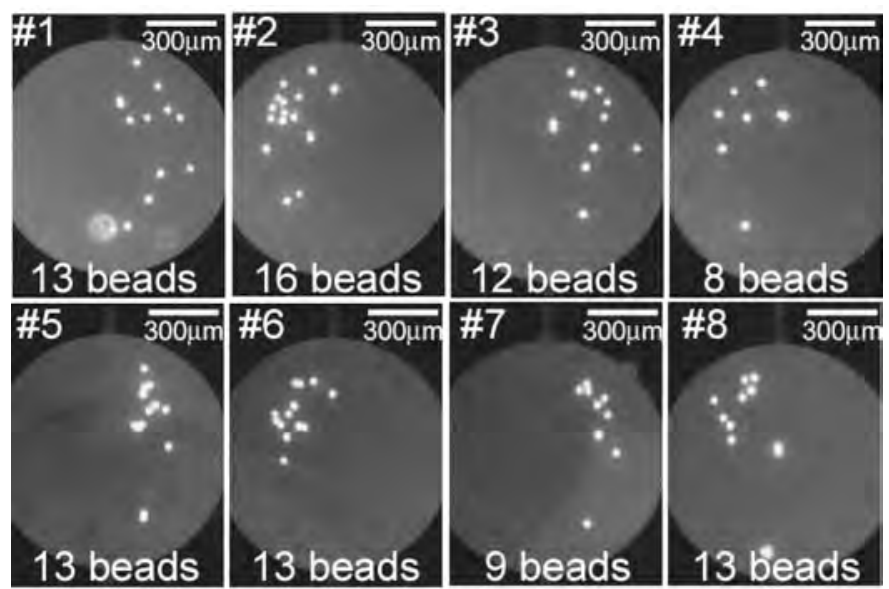

Fig. 6. Fluorescent images of the beads distributed into the 8 microwells of the prototype device. Average number of beads per microwell was 12.5 .

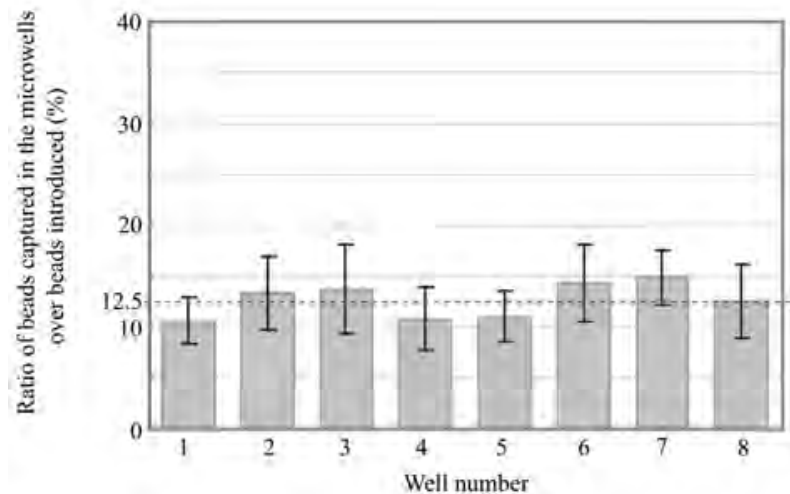

Fig. 7. Bead distribution ratio in the 8 microwells of the prototype device, averaged over 10 measurements. Mean is $12.5 \%$.

inhomogeneous distribution of the cells in the microwells. Adjusting the channel width can effectively reduce the problem of clogging. From our experimental results, the channel width should be at least double the size of the sample diameter (include contamination). For example, at a minimum velocity of $0.1 \mu \mathrm{L} / \mathrm{min}$, polystyrene beads of $20 \mu \mathrm{m}$ diameter did not absorb or clog in a channel of $50 \mu \mathrm{m}$ width. Treatment of the channel surface is also recommended to prevent the absorption of biological substances. For practical use, both the density of the cell suspension and the flow rate at which the cell suspension is introduced must be optimized. When a fluorescent bead suspension with higher density was introduced, the stacking of beads was observed at the pillar colonnades in the microchannel. A density of less than $1.0 \times 10^{3}$ cells $/ \mu \mathrm{L}$ is preferable. 
It is also preferable that the cell suspension flow rate is less than $0.1 \mu \mathrm{L} / \mathrm{min}$ at each microwell. At a flow rate higher than $0.4 \mu \mathrm{L} / \mathrm{min}$ in the microwell, all the fluorescent beads are removed from the microwells. Cell introduction and purging can be realized by simply controlling the flow rate.

\section{Conclusions}

To improve the uniformity of the number of beads passively distributed in a highthroughput cellular analysis device, we introduced the concept and developed the method of self-regulating pinched flow. Using CFD simulation, we optimized the structure of the pinching area. The results of experiments using fluorescent beads showed that the CV of the average of eight microwells improved from 94.9 to 29.3\%. The cells were introduced into the microwells only at a low flow rate; however, after the reagents were injected, the stimulated cells were easily collected at a high flow rate. The number of microwells can be increased simply by adding bifurcations. We expect that this concept and method will be useful for drug screening or toxicity testing in the field of drug discovery.

\section{Acknowledgements}

This work was partially supported by a Japan Ministry of Education, Culture, Sports Science \& Technology Grant-in-Aid for COE Research and 21st COE of Waseda University, Scientific Basic Research (A) No. 12450167, and by a Japan Society for the Promotion of Science Grant-in-Aid for Creative Scientific Research No. 13 GS0024.

\section{References}

1 M. Kanai, H. Abe, T. Munaka, Y. Fujiyama, D. Uchida, A. Yamayoshi, H. Nakanishi and S. Shoji: Sens. Actuators, A 114 (2004) 129.

2 M. Kanai, T. Munaka, H. Abe, Y. Fujiyama, D. Uchida, H. Mikado, H. Nakanishi and S. Shoji: Proc. Int. Conf. Miniaturized Systems Chemistry Life Sciences, $\mu$ TAS 2004 (RSC, London, 2004) p. 126.

3 P. Telleman, U. D. Laesen, J. Phillip, G. Blankenstein and A. Wolff: Proc. Int. Symp. Micro Total Analysis Systems, $\mu$ TAS'98 (Kluwer Academic, Dordrecht, 1998) p. 39.

4 S. Otsuka, M. Kanai, M. Hayashi, H. Nakanishi and S. Shoji: Proc. Int. Conf. Miniaturized Systems Chemistry Life Sciences, $\mu$ TAS 2004 (RSC, London, 2004) p. 30

5 K. Tashiro, S. Ikeda, T. Sekiguchi, H. Sato, S. Shoji, H. Makazu, K. Watanabe, T. Funatsu and S. Tsukita: Tech. Dig. Int. Conf. Solid-State Sensors Actuators Microsystems, Transducers'01 (Springer, Berlin, 2001) p. 932.

6 T. Hara, T. Ichiki, Y. Horiike and K. Yasuda: Proc. Int. Conf. Miniaturized Chemical Biochemical Analysis Systems, TTAS 2002 (Kluwer Academic, Dordrecht, 2002) Vol. 1, 124.

7 D. Holmes, M. E. Sandison, N. G. Green and H. Morgen: Proc. Int. Conf. Miniaturized Systems Chemistry Life Sciences, $\mu$ TAS 2004 (RSC, London, 2004) p. 6.

8 R. Gomez-Sjoberg, AA. Leyrat, DM. Pirone, CS. Chen and SR. Quake: Anal. Chem. (2007) 10.1021/ac071311w.

9 K. Kawai, M. Kanai, H. Nakanishi and S. Shoji: J. Appl. Phys. 45 (2006) 5607.

10 Y. Sai, M. Yamada, M. Yasuda and M. Seki: Proc. Int. Conf. Miniaturized Systems Chemistry Life Sciences, $\mu$ TAS 2005 (RSC, London, 2005) p. 346. 